contract/indemnity/insurance issues. General Practitioners felt that the General Practitioner in/alongside Emergency Department role should be supported professionally e.g. by the Royal Colleges. Finally, General Practitioners were concerned that employing General Practitioners in Emergency Departments may have the unintended consequence of depleting primary care general practice.

Conclusion General Practitioners' perspectives of General Practice Services in or alongside Emergency Departments identify systems and individual benefits and issues which may be used by policy makers and managers when planning and implementing General Practitioner Services in/alongside Emergency Departments.

\section{OP86 USING NORMALISATION PROCESS THEORY TO EXPLORE THE IMPACT OF GENERAL PRACTITIONERS WORKING IN OR ALONGSIDE EMERGENCY DEPARTMENTS IN ENGLAND: QUALITATIVE FINDINGS FROM A NATIONAL MIXED METHODS EVALUATION}

${ }^{1}$ Arabella Scantlebury* ${ }^{1}$ Joy Adamson, ${ }^{1}$ Helen Anderson, ${ }^{1}$ Heather Leggett, ${ }^{2}$ Sarah Voss, ${ }^{2}$ Heather Brant, ${ }^{3}$ Jonathan Benger. ${ }^{1}$ York Trials Unit, Department of Health Sciences, University of York, York, UK; ${ }^{2}$ Faculty of Health and Life Sciences, University of the West of England, Bristol, UK; ${ }^{3}$ Bristol NHS Clinical Commissioning Group, Bristol NHS Clinical Commissioning Group, Bristol, UK

\subsection{6/jech-2021-SSMabstracts.86}

Background It is estimated between $15 \%$ and $40 \%$ of patients attending emergency departments could be treated in General Practice (GP). Hospitals throughout England have introduced GP services in or alongside emergency departments (GPED) to try to reduce demand. Our aim was to explore the impact of GPED and the extent to which it has become a part of routine practice.

Methods Qualitative study consisting of: non-participant observation of 142 individual clinical encounters and 413 semistructured interviews with key stakeholders (policymakers, service leaders, ED staff, GPs, patients and carers). This was distributed across 64 NHS emergency departments in England, including 10 case sites. A coding framework was formed with patient collaborators and used with the pen portrait method for case sites. Findings were mapped onto the four main constructs of NPT: coherence, cognitive participation, collective action and reflexive monitoring.

Results There was widespread disagreement at individual, stakeholder and organisational levels regarding the purpose and potential impact of GPED (Coherence). Participants criticised policy development and implementation and viewed it as a rushed, top-down generalised solution to local problems. The amount of 'work' staff were willing to invest into GPED was hindered by tensions between ED and GP staff; often stemming from different attitudes to risk, perceptions of the GPED GP role and of what should be considered a GPED appropriate patient (Cognitive participation). Streaming and implementation issues (e.g. interprofessional relationships and structural support) and staffing and resource constraints affected how staff used GPED and the extent that it was embedded into routine practice. Concerns that GPED may encourage patients to attend ED and strong views around 'appropriate' ED attendance also influenced how staff and patients viewed GPED (Collective action). There was a lack of consensus as to whether GPED could be considered a success, due to variations in GPED model, site-specific patient mix and governance arrangements (Reflexive monitoring).

Conclusion Translating policy into practice is complex. Our findings highlight challenges of applying a national policy locally. We identified a series of success factors (e.g. inter-professional working, leadership, staffing) for introducing GPED, a number of which are commonly cited as barriers/enablers for introducing health policy in the wider literature.

\section{OP87 CO-DESIGNING A DEEP END GP NETWORK FOR THE NORTH EAST AND NORTH CUMBRIA (NENC)}

Claire Norman*, Josephine M Wildman, Sarah Sowden. Population Health Sciences Institute, Newcastle University, Newcastle Upon Tyne, UK

\subsection{6/jech-2021-SSMabstracts.87}

Background From their Scottish origins in 2009, Deep End GP networks are being established all over the UK and further afield in Ireland and Australia. Formed of primary care practitioners in areas of high blanket socioecocomic deprivation, their common goal it to mitigate health inequalities and champion the cause of primary care. As the North East is the most deprived region in England, it was important to set up a network that was sustainable and reflected the priorities of those who worked in it. The network currently consists of the 34 most deprived practices in the region. Deep End NENC is affiliated with the Newcastle University Applied Research Collaboration's (ARC) 'inequalities and marginalised communities' strand and this work will also be used to direct the ARC research priorities. This project aims to use co-design methodology to gather information from practitioners in the region that could be used to guide the initial steps of the Deep End NENC network. Co-design interviews would also serve to improve engagement and disseminate information about the network.

Methods Participants were recruited using purposive and snowball sampling, as well as a blanket communication to all Deep End practices. Fifteen semi-structured interviews were carried out with health professionals (11 GPs, 2 social prescribing link workers, 1 nurse practitioner and 1 district nurse) from Deep End practices in the NENC. Due to Covid19 these were carried out over Zoom before transcription and thematic analysis. Findings from the interviews were communicated to the members of Deep End NENC via webinars and they also formed the part of the Deep End Steering Group spending prioritisation protocol.

Results A coding framework was used to consider findings at patient level, practice/network level and 'upstream' political level. Themes identified were the specific clinical and social challenges in the Deep End; barriers to patient care and access to services; training and recruitment; the need to connect with others who worked in these communities to share best practice; and the need to advocate for the Deep End. The COVID-19 pandemic itself brought challenges that were felt more acutely by Deep End patients and those who cared for them.

Discussion These interviews were successful at identifying priority areas that will form the basis of the work that Deep End NENC will focus on over the coming years. They also add to the literature around challenges facing staff who work in deprived communities. 